Research paper

\title{
Validation of a rapid antigen test as a screening tool for SARS-CoV-2 infection in asymptomatic populations. Sensitivity, specificity and predictive values
}

\author{
Alejandro Fernandez-Montero ${ }^{\mathrm{a}, \mathrm{b}, *}$, Josepmaria Argemi $^{\mathrm{b}, \mathrm{c}}$, José Antonio Rodríguez ${ }^{\mathrm{b}, \mathrm{d}, \mathrm{e}}$, \\ Arturo H. Ariñof, Laura Moreno-Galarraga ${ }^{\text {b,g,h }}$ \\ a Department of Occupational Medicine, University of Navarra, Av. Pío XII, 36, Pamplona, Spain \\ ${ }^{\mathrm{b}}$ IdiSNA (Instituto de Investigación Sanitaria de Navarra), Pamplona, Spain \\ ' Liver Unit, Clinica Universidad de Navarra, Hepatology Program, CIMA-Universidad de Navarra, Pamplona, Spain \\ ${ }^{\mathrm{d}}$ Atherothrombosis Laboratory, Program of Cardiovascular Diseases, CIMA-Universidad de Navarra, Pamplona, Spain \\ e CIBERCV, ISCIII, Madrid, Spain \\ ${ }_{\mathrm{f}}^{\mathrm{f}}$ Department of Environmental Biology, University of Navarra, Pamplona, Spain \\ ${ }^{\mathrm{g}}$ Department of Pediatrics, Complejo Hospitalario de Navarra, Servicio Navarro de Salud, Pamplona, Spain \\ ${ }^{\mathrm{h}}$ Department of Preventive Medecine and Public Health, Universidad Publica de Navarra, Pamplona, Spain.
}

\section{A R T I C L E I N F O}

\section{Article History:}

Received 7 April 2021

Revised 14 May 2021

Accepted 20 May 2021

Available online 9 June 2021

\section{Keywords:}

SARS-CoV-2

COVID-19

Rapid antigen detection

RT-PCR

Screening tool

\begin{abstract}
A B S T R A C T
Background: Early diagnosis of SARS-CoV-2 infection is essential to reduce disease spread. Rapid antigen tests have not been sufficiently evaluated in asymptomatic patients to be used as massive population screening tools.

Methods: Head-to-head evaluation of Roche SARS-CoV-2 Rapid Antigen Test and real-time reverse transcription polymerase chain reaction (RT-PCR) as SARS-CoV-2 screening tools performed in asymptomatic adults from a semi-closed community in University of Navarra (Spain) from November 2020 to January 2021. Sensitivity, specificity and predictive values were calculated using RT-PCR as reference method.

Findings: Roche SARS-CoV-2 Rapid Antigen Test was performed on 2542 asymptomatic adults in a community with a SARS-CoV-2 incidence of $1.93 \%$. It showed a sensitivity of $71.43 \%$ (CI 95\%: $56.74-83.42$ ) and a specificity of 99.68\% (CI 95\%: 99.37 - 99.86). Positive Predictive Value was 81.4 (CI 95\% 66.6 - 91.61) and Negative Predictive Value was 99.44 (CI 95\% 99.06 - 99.69). Test sensitivity was related to viral load, with higher sensitivity in RT-PCR cycle threshold (Ct) values under 25 (93.75\%, CI 95\%: 71.96 - 98.93), that dropped to $29.41 \%$ (CI 95\%: 10.31- 55.96) in RT-PCR Ct values above 25.

Interpretation: This study suggests that rapid antigen tests are less effective in asymptomatic population, when compared with RT-PCR. Further studies are needed to evaluate different options to improve screenings based on rapid antigen test, such as the use of clinical questionnaires to select higher risk-participants, the confirmation of negative results with RT-PCR or the use of repetitive sequential testing. Funding: This research received no external funding.
\end{abstract}

(c) 2021 The Author(s). Published by Elsevier Ltd. This is an open access article under the CC BY-NC-ND license

(http://creativecommons.org/licenses/by-nc-nd/4.0/)

\section{Introduction}

The search for rapid, accurate and affordable Severe Acute Respiratory Syndrome Coronavirus 2 (SARS-CoV-2) diagnosis tools has become a global priority during Coronavirus-related Disease (COVID19) pandemic. Effective tests useful for widespread testing and

Brief title: Validation of SARS-CoV-2 rapid antigen test in asymptomatic.

* Corresponding author at: Department of Occupational Medicine, University of Navarra, Av. Pío XII, 36, Pamplona, Spain.

E-mail address: afmontero@unav.es (A. Fernandez-Montero). contact tracing are essential in public health policies to control COVID-19 [1-3].

The real-time reverse transcription polymerase chain reaction (RT-PCR) obtained from nasopharyngeal or oropharyngeal swab samples is currently the most used diagnosing-test and it is considered the gold standard specimen for SARS-CoV-2 RNA testing [4-6]. It is a very sensitive method and when used quantitatively, analyzing the cycle threshold $(\mathrm{Ct})$, it also gives information on the viral load and the infectiousness [7]. However, sometimes its use is limited, as it requires long turnaround times, skillful operators, biosafety measures, expensive instrumentation and a laboratory setting, resources not always available in most geographical areas. 


\section{Research in context}

\section{Evidence before this study}

The pandemic caused by the Severe Acute Respiratory Syndrome Coronavirus 2 (SARS-CoV-2) is causing severe health, social and economic problems worldwide. The search for rapid, accurate and affordable SARS-CoV-2 diagnosis tools has become a global priority. The real-time reverse transcription polymerase chain reaction (RTPCR) is a very sensitive method but Rapid diagnosis tests, based on antigen detection are faster, easier to perform and cheaper. There is scarce evidence on the accuracy of these tests in widespread testing of asymptomatic populations in different settings. Krüttgen et al and Lefever et al in 2021 have performed some previous validation study, but in smaller samples or including symptomatic patients.

\section{Added value of this study}

It is urgent to validate SARS-CoV-2 rapid antigen tests for widespread SARS-CoV-2 testing, for the diagnosis of SAR-CoV-2 infection in asymptomatic patients. Our results with Roche rapid antigen test on 2542 asymptomatic adults showed a sensitivity of $71.43 \%$ (CI 95\%. $56.74-83.42$ ) and a specificity of $99.68 \%$ (CI 95\%: 99.37 - 99.86). Test sensitivity was related to viral load, with higher sensitivity in RT-PCR Ct values under 25.

\section{Implications of all the available evidence}

This study demonstrates that rapid antigen tests are less effective in asymptomatic population. Further strategies such as periodic sampling or repeated measures might increase the test efficacy and should therefore be evaluated.

Rapid diagnosis tests based on antigen detection are faster, easier to perform and cheaper. They can be used outside the laboratory and with fast turnaround times, therefore they could be essential when implementing population policies. These rapid tests, through a rapid chromatographic immunoassay technique, are used for the qualitative detection of SARS-CoV-2 antigens generally in nasopharyngeal swabs, they can be easily interpreted without specialized instrument and in less than 30 min. Most rapid antigen test have been designed and validated for the detection of SARS-CoV-2 in patients with COVID-19 symptoms in hospital settings, and there is currently little evidence on the accuracy of these tests in widespread testing of asymptomatic populations and in different settings [8].

According to the WHO, these rapid test need to be better evaluated and they are currently not recommended for clinical diagnosis [9]. As recently published by Boehme et al. generalized testing, outside a health-care setting, depends on factors related to the test, such as test-sensitivity, sampling method, turnaround-time and costs, and on factors related to the setting or community where the test will be used, such as level of exposure, contact patterns, local prevalence and type of community [1]. During the COVID19 pandemic, public health policies aiming at keeping the regular activity in semi-closed communities, like workplaces, schools or universities, are based on general measures, such as social distancing, mask wearing and basic hygienic measures, but a proactive broad population surveillance to stop asymptomatic spread and prevent outbreaks is also advisable and has been implemented in many regions. Nevertheless, head-to-head comparisons of available SARS-CoV-2 rapid antigen detection tests are scarce, with limited sample-size and wide range of clinical settings showing irregular test-performance between different studies and different brands [10,11].

The objective of this study was to validate a SARS-CoV-2 rapid antigen test for widespread SARS-CoV-2 testing in a semi-closed community. We present the results of a single-center cross-sectional head-to-head validation study of Roche SARS-CoV-2 Rapid Antigen Test for the diagnosis of SAR-CoV-2 infection in asymptomatic adults from a university community, using RT-PCR test as the reference standard.

\section{Methods}

\subsection{Population}

Study performed within the COVID-19 transmission prevention plan of the University of Navarra (Pamplona, Spain) where massive randomized SARS-CoV-2 PCR tests are periodically performed. The present study was carried out according to STARD guidelines among randomly selected university students and employees. Inclusion in the study was voluntary and informed consent was signed prior to inclusion. Out of the 16,869 potentially eligible participants (current staff and students from the University of Navarra), 2543 were included in this study after being scheduled for a randomized mass testing between November 2020 and January 2021, as shown in Fig. 1. The only exclusion criterion was the presence of COVID-19 symptoms (fever, persistent cough, or shortness of breath). Patients with nonspecific mild symptoms (such as nasal congestion, odynophagia, isolated headache or isolated gastrointestinal symptoms) and COVID-19 close-contacts (individuals who had been in contact with a Covid-19 positive patient without adequate protection measures, closer than $2 \mathrm{~m}$ and for longer than $15 \mathrm{~min}, 48 \mathrm{~h}$ before symptoms onset or diagnosis of the Covid-19 patient) were not excluded.

\subsection{SARS-CoV-2 testing}

Nasopharyngeal and oropharyngeal swabs samples were obtained from each participant by a trained nurse on the same visit. The rapid antigen test and the RT-PCR for SARS-CoV-2 detection, were simultaneously performed.

Roche SARS-CoV-2 Rapid Antigen Test is a rapid chromatographic immunoassay intended for the qualitative detection of specific antigens of SARS-CoV-2 present in human nasopharynx. It detects the SARS-CoV-2 nucleoprotein, and it can be used to assess whether a person is infected with SARS-CoV-2 by providing a qualitative result shown as colored bands. In addition, the SARS-CoV-2 Rapid Antigen Test does not use any laboratory instruments that automate the process, it is therefore easy to use, and it does not require specific training. The detailed test protocol as well as the validation results (sensitivity 96.52\% and specificity 99.68\%) in hospital setting can be found on the manufacturer's website [12]. The rapid antigen test was performed by trained personnel on nasopharyngeal samples on site, following the manufacturer's instructions. Two technicians performed two independent blind readings. When a disparity between readings appeared $(0.32 \%)$, a third technician broke the tie.

RT-PCR was carried out in the Genomic Unit of the Center for Applied Medical Research (CIMA) at the University of Navarra. Samples were obtained by oropharyngeal swabbing and placed in a polypropylene tube with $500 \mu \mathrm{l}$ of lysis solution (RLT Plus, Qiagen). Samples were then transferred from the tubes to a 96-well plate using a Tecan Freedom EVO 150 robotic system. RNA extraction was carried out according to the protocol developed by CIMA researchers [13] using superparamagnetic particles (Dynabeads MyOne SILANE, Thermofisher), adapted for automation on an Agilent Bravo NGS system. Briefly, the samples were mixed with the magnetic particles and ethanol, so that the particles capture the RNA [14]. The magnetic particles are then immobilized with a magnet, washed twice with $80 \%$ ethanol and nucleic acids are eluted in $10 \mathrm{mM}$ Tris, $\mathrm{pH}$ 7. The "Realtime fluorescent RT-PCR kit for detecting 2019-nCoV" (BGI ref. HW4412) was used for the detection of coronavirus RNA by reverse transcription quantitative PCR (RT-qPCR) in a thermal cycler 


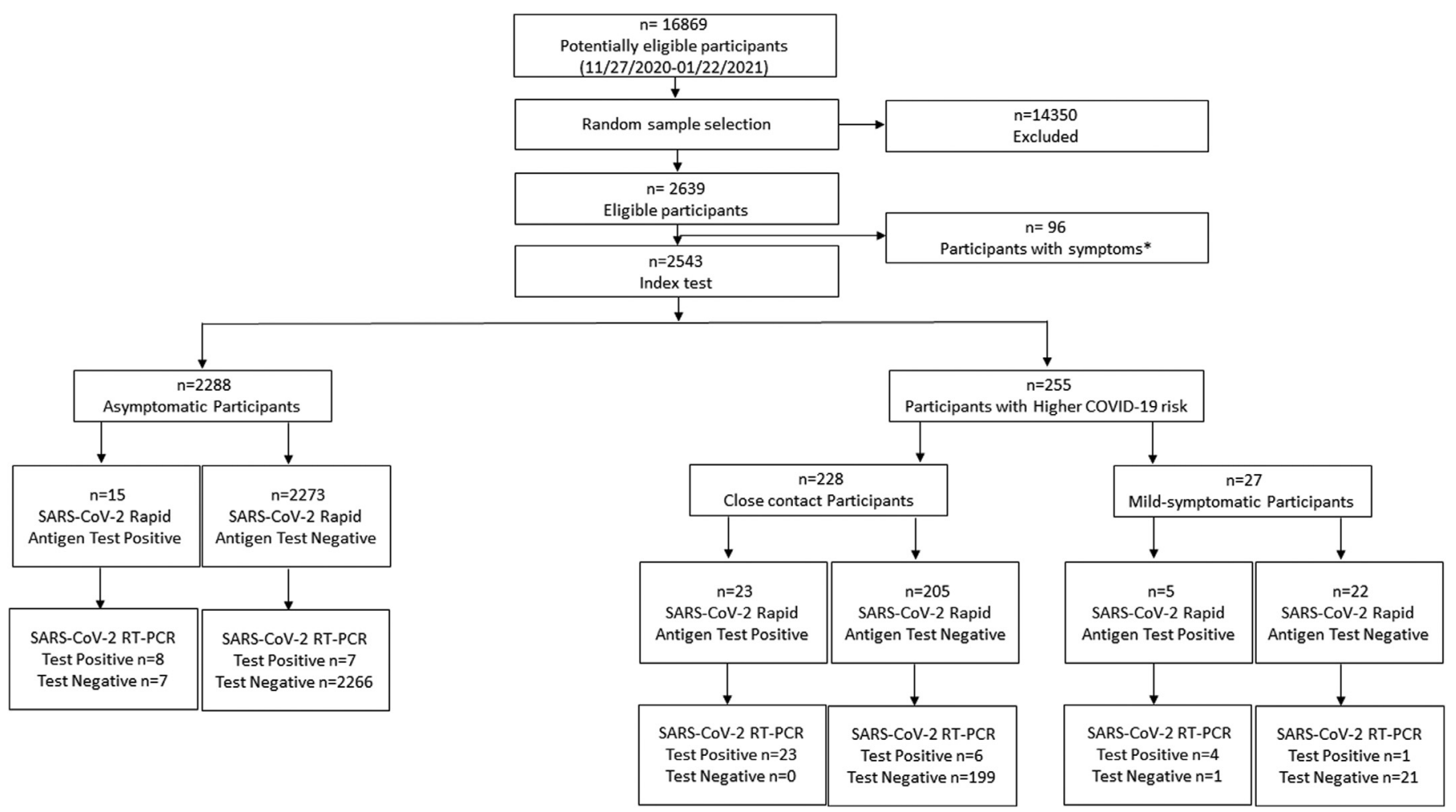

Fig. 1. Study Flow-chart: Validation of a rapid antigen test as a screening tool for SARS-CoV-2 infection in asymptomatic populations. * Fever, persistent cough, or shortness of breath.

(QuantStudio 5, Thermofisher). Results were then analyzed with QuantStudio Design Analysis Software (Thermofisher). This process has been validated by the Spanish Carlos III Health Institute in April 2020.

Samples for RT-PCR analysis were transferred to the laboratory in the next $5 \mathrm{~h}$ after collection. Samples were stored at local room temperature. The CIMA analyzed and reported the results on the same day the sample was collected. The results of both tests were independently introduced into independent databases, anonymized and merged into a single database for analysis.

\subsection{Statistical analysis}

Sensitivity, specificity, negative predictive value (NPV) and positive predictive value (PPV) with their respective 95\% confidence intervals $(\mathrm{CI})$ using binomial or Agresti and Coull methods. Analyses were performed on the total sample and splitting the population into two categories: "asymptomatic low-risk participants" and "participants at increased risk of SARS-CoV-2 infection" (including COVID-19 close contacts and those who reported non-specific mild symptoms). Kappa index was used to study tests correlation.

Based on previous studies and in order to be able to compare our results with previous findings, the positive RT-PCR test results have been categorized and analyzed according to their quantitative Ct-value in two different ways. First using the Ct-values divided into four intervals $(<20,20$ to $<25,25$ to $<30$, and $\geq 30 \mathrm{Ct})$ and later using four accumulative categories $(<20,<25$, $<30$ and $<35$ ). Rapid antigen test sensitivity was calculated across $\mathrm{Ct}$ threshold categories.

A bee swarm style graphic representation of the positive RT-PCR tests according to their Ct-value and divided into two groups depending on whether the rapid antigen test had been positive or negative was also performed. On this graph the median $\mathrm{Ct}$ value is represented by the middle line in each swarm while the upper and lower lines represent the interquartile range (p75 and p25). A Mann-Whitney U test was performed to compare the differences among groups. Analyses were performed using STATA 12.0 .

Sample size estimation was determined considering a power of 0.90 , a type I error of 0.05 and a SARS-CoV-2 prevalence of $1.3 \%$. Sensitivity values and specificity of 96.52 and 99.68 respectively were assumed as previously reported in clinical setting.[12] The estimated sample size for rejecting the null hypothesis of non-inferiority to PCR testing was 760 participants. Missing data and dropout rate were zero, recruitment coincided with the first and only visit and all randomly selected participants accepted to enter the study.

Roche Diagnostics International Ltd. provided the rapid antigen tests required for the study. A contract was signed with Roche Diagnostics International Ltd. including our commitment to perform the test according to their protocols and defining the role of the funding source. The contract specified that Roche will not be involved in the study-design, data-analysis nor in the interpretation of results or in the decision to publication, and that Roche will have access to the study results, but not to the study data. The study was conducted in compliance to the study protocol, the current version of the Declaration of Helsinki, and the local, legal and regulatory requirements (Approved by the University of Navarra Ethics committee: 2020.216 on October 30th, 2020). An informed consent was given to all participants and singed by both parties.

\subsection{Role of the funding source}

This research received no external funding. Roche Diagnostics International Ltd. donated the rapid antigen tests required for the study, but had no role in data collection, analysis, generation of results or decision to submit for publication. Dr. Fernandez-Montero, Prof. Ariño and Dr. Moreno-Galarraga had access to the full dataset and took the decision to submit the manuscript for publication. 
Table 1

Baseline characteristics of participants according to risk of SARS-CoV-2 infection.

\begin{tabular}{lllr}
\hline & Asymptomatic & Higher COVID-19 risk $^{*}$ & $\mathrm{p}$ \\
\hline $\mathrm{N}$ & 2288 & 255 & \\
Sex, women N (\%) & $1392(62.06)$ & $165(65.74)$ & 0.254 \\
$\begin{array}{l}\text { Age, mean (Standard } \\
\quad \text { deviation) }\end{array}$ & $28.06(12.32)$ & $23.63(7.1)$ & $<0.001$ \\
\hline
\end{tabular}

*Including participant with non-specific mild symptoms and close contacts.

\section{Results}

Out of 2543 participants (62.4\% females, mean age $27.61 \pm 11.97$ year-old), 49 cases of SARS-CoV-2 infection were RT-PCR confirmed during the study period. Sample description is showed in Table 1. Total SARS-CoV-2 incidence was $1.93 \%$. The study sample was mainly composed of asymptomatic participants (90\%) among whom COVID19 incidence was $0.66 \%$. The rest of participants were considered as higher COVID-19 risk participants, and this group included close COVID-19 contacts (9\%) with a $12.72 \%$ incidence, and mild-symptomatic participants (1\%) with a $18.52 \%$ incidence of COVID-19. No adverse events were detected during the procedures.

The rapid SARS-CoV-2 antigen test showed in the total sample low sensitivity (71.43\%), moderate PPV (81.40\%), high specificity (99.68\%) and high NPV (99.44\%) (Table 2) with a moderate agreement (kappa index $=0.76$ ) between the two tests. When the sample was divided into asymptomatic-low-risk and high-risk participants, the rapid SARS-CoV-2 antigen test presented lower sensitivity (53.33\%) and PPV (53.33\%) values and higher specificity (99.69\%) and NPV (99.69\%) in asymptomatic participants, and moderate amounts of sensitivity (79.41\%), high specificity (99.55\%), PPV (96.43\%) and NPV (96.91\%) in patients with higher COVID-19 risk (Table 2).

Table 3 shows how the rapid SARS-CoV-2 antigen test has a high capacity to detect positives in samples with higher viral loads (Sensitivity of 93.75\% (CI 95\% 79.19-99.23) in RT-PCR Ct values below 25) but a lower capacity to detect infection in samples with lower viral load (Sensitivity of 29.41\% (CI 95\% 10.31-55.96) in RT-PCR Ct values above 25). When analyzing the results dividing the sample in four accumulative categories, sensitivity ranged from $100 \%$ in $\mathrm{Ct}<20$ to $72.92 \%$ in $\mathrm{Ct}<35$. A decline in the test performance was observed as the $\mathrm{Ct}$ values increased (Table 4).

Fig. 2 shows significant difference in the Ct values when comparing the group with positive RT-PCR and negative Rapid Antigen Test (median Ct values 29.95 interquartile range 28.07-32.05) with the group with positive RT-PCR and positive Rapid Antigen Test (median Ct values 22.3 interquartile range $19.91-24.42)(p<0.001)$.

\section{Discussion}

This observational study, designed to analyze the efficacy of the Roche SARS-CoV-2 Rapid Antigen Test, showed the limitations of this test when used as a screening massive tool for the diagnosis of acute SARS-CoV2 infection in asymptomatic populations.
Table 3

Sensitivity of the SARS-CoV-2 Rapid Antigen Test according to RT-PCR Ct intervals.

\begin{tabular}{lllc}
\hline Ct values & $\mathrm{N}$ & True positive & Sensitivity\%(CI95\%) \\
\hline$<20$ & 9 & 9 & $100(65.54-100)^{*}$ \\
$20-<25$ & 23 & 21 & $91.30(71.96-98.93)$ \\
$25-<30$ & 9 & 4 & $44.44(13.7-78.8)$ \\
$\geq 30$ & 8 & 1 & $12.5(0.32-52.65)$ \\
\hline * calculate Agresti-Coull confidence intervals.
\end{tabular}

\section{Table 4}

Sensitivity of the SARS-CoV-2 Rapid Antigen Test according to RT-PCR Ct accumulative categories.

\begin{tabular}{lllc}
\hline Ct values & $\mathrm{N}$ & True positive & Sensitivity\%(CI95\%) \\
\hline$<20$ & 9 & 9 & $100(65.54-100)^{*}$ \\
$<25$ & 32 & 30 & $93.75(79.19-99.23)$ \\
$<30$ & 41 & 34 & $82.93(67.94-92 \cdot 85)$ \\
$<35$ & 48 & 35 & $72.92(58.15-84.72)$ \\
\hline * calculate Agresti-Coull confidence intervals.
\end{tabular}

It is urgent to validate the new rapid diagnostic techniques to detect SARS-CoV2 infection status in different clinical settings and in different populations. Screening programs are essential in public health strategies and massive testing is essential as social distancing, lock downs and home confinement cannot be long term measures. Tests used in these screening programs should be validated in different settings and conditions, to optimize the available test according to each context. The purpose of this study was to validate a rapid antigen test as a screening tool in a semi-closed community, (University community) in a pandemic situation (incidence $1.93 \%$ ) evaluating its suitability for diagnosing acute infection in asymptomatic adults. When used on symptomatic patients Roche SARS-CoV-2 rapid antigen test has proven to have high sensitivity and specificity [12], meeting WHO recommended criteria of $\geq 80 \%$ sensitivity and $\geq 97 \%$ specificity. The results of the Roche-rapid test on this low-prevalent population when compared with RT-PCR test showed a sensitivity of $71.43 \%$ and a specificity of $99.68 \%$ with a moderate concordance between both tests $(k=0.76)$. These results were comparable to those recently obtained in studies based on rapid antigen detection tests, in mixed populations including both symptomatic and asymptomatic individuals $[11,15]$. We found, as previously published $[15,16]$, that rapid antigen test sensitivity was related to viral load, showing better results in samples with lower RT-PCR Ct values. Krüttgen et al. calculated that the Roche SARS-CoV-2 Rapid Antigen Test sensitivity was $100 \%$ in samples with a high viral load $(\mathrm{Ct}<25)$ but that the test sensitivity dropped to $95 \%, 44.8 \%$, and $22.2 \%$ in samples with medium (Ct $25-<30$ ), low (Ct 30-<35) and very low (Ct $>35$ ) viral loads, respectively [16]. Lefever et al. [17] also validated a rapid-test although in their study they used an automated reading test performed in a laboratory (DiaSorin Liaison antigen test) and used a different genetic target in the RT-PCR test (N1 and N2 region of the nucleocapsid phosphoprotein gene), despite these differences this study showed almost identical results. We performed additional

Table 2

Validation result of SARS-CoV-2 Rapid Antigen Test compared to RT-PCR test.

\begin{tabular}{lccc}
\hline & Asymptomatic & Higher COVID-19 risk* & Total sample \\
\hline $\mathrm{N}$ & 2288 & 255 & 2543 \\
Cases (incidence) & $15(0.66)$ & $34(13.33)$ & $49(1.93)$ \\
Sensitivity\% (IC95\%) & $53.33(26.59-78.73)$ & $79.41(62.10-91.30)$ & $71.43(56.74-83.42)$ \\
Specificity\% (IC95\%) & $99.69(99.37-99.88)$ & $99.55(97.50-99.99)$ & $99.68(99.37-99.86)$ \\
Positive predictive value\%(CI95\%) & $53.33(26.59-78.73)$ & $96.43(81.65-99.91)$ & $81.40(66.60-91.61)$ \\
Negative predictive value\%(CI95\%) & $99.69(99.37-99.88)$ & $96.91(93.74-98.75)$ & $99.44(99.06-99.69)$ \\
\hline
\end{tabular}

*Including participant with non-specific mild symptoms and close contacts. 


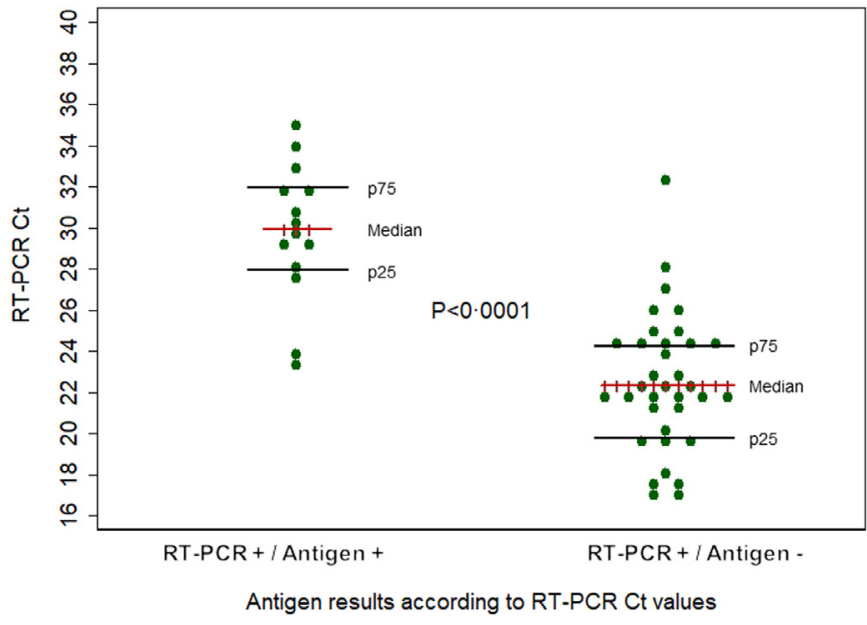

Fig. 2. Graphical representation of positive and negative antigen results according to RT-PCR Ct values. Validation study of a rapid antigen test as a screening tool for SARSCoV-2 infection.

RT-PCR: Real-time reverse transcription polymerase chain reaction; $\mathrm{Ct}$ : cycle threshold.

analysis, calculating the different levels of sensitivity across CT categories as performed in the work published by Lefever et al. When using the same CT cut-off threshold in our study, (cut-off points of $<20,<25,<30$ and $<35$ ), we appreciate a sensitivity of $100 \%, 93.8 \%$, $82.9 \%$ and $72.92 \%$ respectively, very similar to the data published by Lefever et al. showing a sensitivity of $100 \%, 94.8 \%, 83.2 \%$ and $71.3 \%$. Both studies show how regardless the type of antigen test used, the automation of the process, or the genetic target used in the RT-PCR test, rapid antigen tests decrease their sensitivity to detect SARSCoV-2 as viral load decreases.

As it is not possible to differentiate whether low viral loads are residual viral loads (reflecting the last stage of the disease) or they represent a very early stage of the disease, the false negatives of this study could leave undetected some SARS-CoV2 individuals during the incubation period. This could have dangerous consequences due to a false safety impression on a patient entering a high contagious phase. As samples obtained on the first days after symptoms onset are most likely to contain[18] higher viral loads, this finding is concordant to the current WHO guidelines [19], that recommends that rapid antigen test should be used only in symptomatic cases ( $24 \mathrm{~h}$ after symptoms on-set) and within the first 5-7 days of the onset of symptoms. Being currently crucial to detect asymptomatic early SARS-CoV2 infected subjects, the false-negative antigen tests seen in this cohort $(28,57 \%)$ acquire important public health consequences, as not detecting potentially contagious patients could dangerously increase the infection spread. Roche SARS-CoV-2 Rapid Antigen Test performance has proven to be excellent in nasopharyngeal swabs from symptomatic patients with low $\mathrm{Ct}$ values, but according to the data here presented, it seems to be insufficient for massive screening of asymptomatic population.

The test accuracy on asymptomatic population, might question the value of the rapid antigen test as screening diagnostic tools. Nevertheless, the rapid tests do have an important value when positioned appropriately and they can be useful and efficient tools when used correctly. In a pandemic situation, when a cheap, rapid and widely distributed test may be needed, different options such as using clinical questionnaires to select higher risk patients, the used of repetitive sequential measures [20], or the use of rapid antigen test as an initial screening, understanding that negative results will need to be confirmed by RT-PCR test, will increase the test sensibility. Therefore, rapid antigen tests could be an efficient policy if used correctly. More studies are needed in order to confirm these results and the efficacy and effectivity of repeated testing [21]. In a high prevalence situation, a positive rapid antigen test has a high positive predictive value, whereas negative results should never be used to decrease the standard protective measures.

Our study has some limitations that should be addressed. Sample size calculation was done with an assumption of a $90 \%$ sensitivity based on the manufacturer's information, so the final statistical power was lower than expected. Still, the sample size is large and it triples the required sample size. Viral culture was not performed, so the real relationship between positive rapid antigen test and contagiousness remains unclear. Only RT-PCR Ct values were measured, and although there is significant variation in Ct values depending on the region of the target gene used, they have been related to viral load [22], and furthermore, the results of our study are similar to other studies even when using different target genes $[16,17]$. Also, when designing validation studies, biological, immunologic, technical and temporal aspects are important to consider. The samples used were performed by the same technician and in the same visit, but one test was performed on oropharyngeal swabs while the other was performed on nasopharyngeal swaps which could have led to different RNA concentrations due to different RNA shedding at the two sites. We used the samples required according to each test protocol, but as new screening test are developed, using different samples (nasopharyngeal, oropharyngeal, rectal or saliva) and different collection methods (providervs. patient-collected samples) deserve further study. The evidence regarding different RNA concentrations in different samples and different phase of the infection cycle needs to be better addressed. Finally, another study limitation was the difficulty to differentiate if the low viral-loads undetected by the rapid antigen test (false negatives) represent the initial or the late stage of the disease. If they represent an early moment in the SARS-CoV-2 infection, not detecting these individuals will be a high-risk action, especially if they wrongly get a false feeling of security. On the other hand, if these undetected low viral loads represent a late stage of the disease, individuals will probably be non-infective, or become non-infective soon, and therefore this false negative will not be such a potential theat. We believe further studies, including clinical follow-up and repeated diagnostic test will be key to better understand this situation.

In conclusion, the Roche SARS-CoV-2 Rapid Antigen Test showed a moderate sensitivity on asymptomatic patients, when compared with RT-PCR. Test sensitivity and specificity increased in patients with higher viral loads or higher COVID-19 risk (presence of mild symptoms or known exposure to SARS-CoV-2) but it was lower in asymptomatic low risk patients, with an overall $28.57 \%$ of false negatives.

The Roche SARS-CoV-2 Rapid Antigen Test is not a useful tool to be used as a single screening test in semi-closed low risk communities. Further strategies such as periodic sampling or repeated measures might increase the test efficacy and should therefore be evaluated.

\section{Author contributions: conceptualization}

AFM JA; Data curation: AFM, AHA, and LMG; Formal analysis: AFM; Investigation: AFM and LMG; Methodology AFM and JAR; Supervision: AFM and LMG; Writing-original draft: AFM and LMG; Writing-review \& editing: AFM, AHA, JA, JAR and LMG. All authors have read and agreed to the published version of the manuscript.

\section{Data sharing statement}

Dr. Fernandez-Montero, Prof. Ariño and Dr. Moreno-Galarraga have verified all underlying data. The study data can be requested to Dr. Fernandez-Montero. 


\section{Declaration of Competing Interest}

The authors have no conflict of interest to disclose. Roche Diagnostics International Ltd. provided the rapid antigen tests required for the study. A contract was signed with Roche Diagnostics International Ltd. with the following clauses on dataset and publication: "any Study data generated by the Universidad de Navarra shall be owned by the Institution. Roche had access to study results, but not to study data. After the first submission for publication, Roche may use the Final Study Report for any and all purposes, including but not limited to, research and development, marketing, or future regulatory submissions. A manuscript for publication shall be submitted to Roche for review not later than 180 (one hundred and eighty) days after completion of the Study. Any publications shall comply with applicable laws and guidelines and the recommendations of the International Committee of Medical Journal Editors. Roche will have no role on the decision to submit the paper for publication. The Universidad de Navarra shall safeguard intellectual property rights and provide to Roche the manuscript in its near final form at least 3 months prior to submission to obtain Roche's scientific check as well as to coordinate with Roche the date of publication in order to preclude any preliminary publication which may be detrimental to the novelty of any proposed patent application. Roche's support of the Study shall be disclosed in any Publication, as required by applicable laws, regulations, codes, and guidelines."

\section{Funding}

This research received no external funding.

\section{References}

[1] Boehme C, Hannay E, Sampath R. SARS-CoV-2 testing for public health use: core principles and considerations for defined use settings. Lancet Glob Health 2021;9 (3):e247-9.

[2] Goldstein N, Burstyn I. On the importance of early testing even when imperfect in a pandemic such as COVID-19. Glob Epidemiol 2020;2:100031.

[3] Adalja AA, Toner E, Inglesby TV. Priorities for the US health community responding to COVID-19. JAMA 2020;323(14):1343-4.

[4] National Center for Immunization and Respiratory Diseases (NCIRD) D of VD. Overview of testing for SARS-CoV-2 (COVID-19). Updated 21 Oct[Internet]. CDC.
Available from: https://www.cdc.gov/coronavirus/2019-ncov/hcp/testing-overview.html.

[5] Vashist SK. In vitro diagnostic assays for COVID-19: recent advances and emerging trends. Diagn (Basel) 2020;10(4):202.

[6] Buchta C, Görzer I, Chiba P, et al. Variability of cycle threshold values in an external quality assessment scheme for detection of the SARS-CoV-2 virus genome by RT-PCR. Clin Chem Lab Med 2020 10.1515/cclm-2020-1602.

[7] Bullard J, Dust K, Funk D, et al. Predicting infectious SARS-CoV-2 from diagnostic samples. Clin Infect Dis 2020;71(10):2663-6.

[8] Favresse J, Gillot C, Oliveira M, et al. Head-to-head comparison of rapid and automated antigen detection tests for the diagnosis of SARS-CoV-2 infection. J Clin Med 2021;10(2):265.

[9] WHO. Laboratory testing strategy recommendations for COVID-19: interim guidance. https://apps.who.int/iris/handle/10665/331509 21 March.

[10] Lanser L, Bellmann-Weiler R, Öttl KW, et al. Evaluating the clinical utility and sensitivity of SARS-CoV-2 antigen testing in relation to RT-PCR Ct values. Infection 2020:1-3.

[11] Dinnes J, Deeks JJ, Adriano A, et al. Rapid, point-of-care antigen and molecularbased tests for diagnosis of SARS-CoV-2 infection. Cochrane Database Syst Rev 2021:3 CD013705.

[12] Roche Diagnostics Ltd. SARS-CoV-2 rapid antigen test package insert. 2020

[13] CIMA. Manual silane magnetic bead-based high throughput protocol for SARSCoV-2 RNA extraction V.2 at. 2020 https://www.protocols.io/view/manualsilane-magnetic-bead-based-high-throughput-bfmajk2e.

[14] Berensmeier S. Magnetic particles for the separation and purification of nucleic acids. Appl Microbiol Biotechnol 2006;73(3):495-504.

[15] Mak GC, Lau SS, Wong KK, et al. Analytical sensitivity and clinical sensitivity of the three rapid antigen detection kits for detection of SARS-CoV-2 virus. J Clin Virol 2020;133:104684.

[16] Krüttgen A, Cornelissen CG, Dreher M, Hornef MW, Imöhl M, Kleines M. Comparison of the SARS-CoV-2 rapid antigen test to the real star Sars-CoV-2 RT PCR kit. J Virol Methods 2021;288:114024.

[17] Lefever S, Indevuyst C, Cuypers L, Dewaele K, Yin N, Cotton F, Royal Belgian Society of Laboratory Medicine. Comparison of the quantitative DiaSorin Liaison antigen test to RT-PCR for the diagnosis of COVID-19 in symptomatic and asymptomatic outpatients. J Clin Microbiol 2021;13:00374. 21Epub ahead of print.

[18] Centre for Health Protection. Latest situation of cases of COVID-19. Available at: https://www.chp.gov.hk/files/pdf/local_situation_covid19_en.pdf.

[19] WHO. Antigen-detection in the diagnosis of SARS-CoV-2 infection using rapid immunoassays. Interim Guidance. 11 September.Available at https://www.who. int/publications/i/item/antigen-detection-in-the-diagnosis-of-sars-cov-2infection-using-rapid-immunoassays.

[20] Larremore DB, Wilder B, Lester E, et al. Test sensitivity is secondary to frequency and turnaround time for COVID-19 screening. Sci Adv 2021;7(1):eabd5393.

[21] Viswanathan M, Kahwati L, Jahn B, et al. Universal screening for SARS-CoV2 infection: a rapid review. Cochrane Database Syst Rev 2020;9 CD013718.

[22] Yu F, Yan L, Wang N, et al. Quantitative detection and viral load analysis of SARSCoV-2 in infected patients. Clin Infect Dis 2020;71(15):793-8. 